\title{
Valores Humanos e Bullying: Idade e Sexo Moderam essa Relação?
}

\author{
Renan Pereira Monteiro \\ Universidade Federal do Piauí, Parnaíba, PI, Brasil \\ Emerson Diógenes de Medeiros \\ Universidade Federal do Piauí, Parnaiba, PI, Brasil \\ Carlos Eduardo Pimentel \\ Universidade Federal da Paraíba, João Pessoa, PB, Brasil \\ Ana Karla Silva Soares \\ Universidade Federal do Mato Grosso do Sul, Campo Grande, MS, Brasil \\ Hermógenes Acácio de Medeiros \\ Secretaria Municipal de Saúde de João Pessoa, PB, Brasil \\ Valdiney Veloso Gouveia ${ }^{1}$ \\ Universidade Federal da Paraíba, João Pessoa, PB, Brasil
}

\section{Resumo}

São perceptíveis as consequências graves que perpassam o bullying, sendo importante conhecer variáveis que possam predizer este padrão de comportamento entre escolares. O presente estudo objetivou conhecer em que medida os valores humanos predizem o bullying, testando o papel moderador das variáveis sexo e idade. Participaram 300 crianças $(M=11,07 ; D P=1,31)$ de escolas públicas e particulares, as quais responderam a Escala de Comportamentos de Bullying, o Questionário dos Valores Básicos - Infantil e perguntas demográficas. Os resultados indicaram que os valores das subfunções interativa $(\beta=-0,21, \mathrm{~B}=-0,14$, IC95\% $=-0,23 /-0,06, p<0,001)$ e realização $(\beta=0,32$, $\mathrm{B}=0,14, \mathrm{IC} 95 \%=0,09 / 0,20, p<0,001)$ predisseram comportamentos de bullying. Posteriormente, verificou-se que estas relações não foram moderadas por sexo e idade das crianças. Conclui-se que os valores humanos são uma variável importante para compreender este tipo de comportamento agressivo entre pares, favorecendo intervenções que venham a reduzir este problema nas escolas.

Palavras-chave: Bullying, valores humanos, crianças, escola.

\section{Human Values and Bullying: Do Age and Gender Moderate this Relationship?}

\footnotetext{
Abstract

The serious consequences that underlie bullying are noticeable and it is important to know the variables that can predict this behavior pattern among students. The aim of the study was to understand to what

Endereço para correspondência: Universidade Federal da Paraíba, Centro de Ciências Humanas, Letras e Artes, Departamento de Psicologia, João Pessoa, PB, Brasil 58.051-900. Fone: + 55(83)32167856; Fax: +55(83)32167064. E-mail: vvgouveia@gmail.com

Agradecimentos: Agradecemos à Coordenação de Aperfeiçoamento de Pessoal de Nível Superior (CAPES) e ao Conselho Nacional de Desenvolvimento Científico e Tecnológico (CNPq) em razão de bolsas concedidas ao primeiro (Doutorado em Psicologia Social) e último (Produtividade em Pesquisa) autores.
} 
extent human values predict bullying, testing the moderating role of gender and age. Participants were 300 children $(M=11.07 ; S D=1.31)$ from public and private schools, who answered the Bullying Behaviors Scale, the children version of Basic Values Survey and demographic questions. Results showed that values from the interactive $(\beta=-.21, \mathrm{~B}=-.14$, IC95\% $=-.23 /-.06, p<.001)$ and promotion $(\beta=.32$, $\mathrm{B}=.14$, IC95\% $=.09 / .20, p<.001)$ subfunctions predict bullying behavior. We found that these relationships were not moderated by the children's gender or age. It may be concluded that human values are an important variable to understand this aggressive behavior among peers, promoting interventions that may reduce the problem at schools.

Keywords: Bullying, human values, children, school.

\section{Los Valores Humanos y el Bullying: La Edad y el Género Moderan esta Relación?}

\section{Resumen}

Son perceptibles las graves consecuencias que perpasan el bullying, siendo importante conocer variables que puedan predecir este comportamiento entre estudiantes. El presente trabajo tuvo como objetivo conocer en que grado los valores humanos predicen el bullying, evaluando el papel moderador de las variables género y edad. Participaron 300 niños $(M=11.07 ; S D=1.31)$ de escuelas publicas y privadas, los cuales responderon la Escala de Comportamentos de Bullying, el Cuestionario de Valores Básicos Infantil y preguntas demográficas. Los resultados indicaron que los valores de las subfunciones interactiva $(\beta=-.21, \mathrm{~B}=-.14, \mathrm{IC} 95 \%=-.23 /-.06, p<.001)$ y realización $(\beta=.32, \mathrm{~B}=.14$, IC95\% $=.09 / .20$, $p<.001$ ), predicieron comportamientos de bullying. Posteriormente, se verificó que estas relaciones no fueron moderadas por género y edad de los niños. Se concluye que los valores humanos son una variable importante para comprender este tipo de comportamiento, favorecendo intervenciones que vengan a reducir este problema en las escuelas.

Palabras clave: Bullying, valores humanos, niños, escuela.

Envolver-se em episódios de bullying pode acarretar prejuízos diversos, indo desde ideação suicida e uso de substâncias até retaliações violentas das vítimas (Geel, Vedder, \& Tanilon, 2014; Reed, Nugent, \& Cooper, 2015; Roh et al., 2015). Apesar de tais consequências, são crescentes os relatos de bullying em ambientes escolares, denotando que este tipo de agressão entre pares vem se transformando em um problema de saúde pública (Craig et al., 2009; Hertz, Donato, \& Wright, 2013; Kim, Koh, \& Leventhal, 2005), sendo, portanto, relevantes os intentos de conhecer seus antecedentes.

Em termos conceituais, o bullying configura-se como um subtipo de comportamento agressivo, onde são praticadas, repetidamente e sem justificativa, agressões físicas, verbais e/ou psicológicas, onde o vitimizado se encontra em uma relação desigual de poder, impossibilitando sua defesa (Baldry \& Farrington, 2000; Olweus, 1993; Salmivalli, 2010; Smith \& Morita, 1999). Percebe-se que a intencionalidade do agressor e a assimetria de forças entre perpetrador e vítima são atributos centrais do fenômeno (Farrington, 1993).

Apesar de a literatura apresentar definições e formas específicas de estimar o bullying, a identificação do comportamento correspondente por parte de pais, responsáveis e profissionais ainda é deficitária. De fato, estes agentes comumente caracterizam as agressões sofridas pelas vítimas como parte do processo de socialização, isto é, encara-se o problema como algo natural (Blank \& Liberal, 2005; Lopes, 2005). Tal naturalização do fenômeno contrasta com seus danos severos.

Considerando o anteriormente indicado, há que dizer que entre os participantes dos atos de 
bullying, os agressores (bullies) tendem a apresentar comportamentos antissociais, como o uso de drogas lícitas e ilícitas, além de serem potenciais pessoas em conflito com a lei (Bender \& Lösel, 2011; Farrington \& Ttofi, 2011; Oliveira et al., 2016); as vitimas são propensas a estarem insatisfeitas com a vida, podendo apresentam tendências depressivas e suicidas (Costa, Xavier, Andrade, Proietti, \& Caiaffa, 2015; Fleming \& Jacobsen, 2009; Geel et al., 2014; Klomek, Sourander, \& Gould, 2010; Roh et al., 2015); vitimas/agressores tendem a apresentar baixos índices de comportamento pró-social, frequentes dificuldades acadêmicas e predisposição para o desenvolvimento de transtornos psiquiátricos (Arseneault et al., 2006; Kim et al., 2005; Perren \& Alsaker, 2006; Sourander et al., 2007). Os efeitos do bullying sobre as testemunhas variam de acordo com o envolvimento destes; por exemplo, pode ir da quase extinção de comportamentos relacionados com solidariedade e cooperação até sofrimento vivenciado por aqueles que se identificam com a vítima (Ribeiro, 2007).

Percebe-se, portanto, que a participação em comportamentos de bullying pode trazer prejuízos aos envolvidos, algo que aponta para a necessidade de conhecer variáveis que possam predizê-los. De fato, alguns estudos têm identificado variáveis que podem explicar a predisposição para se envolver em situações de bullying no papel de agressor (e.g., agressividade, raiva, baixa agradabilidade e baixo autocontrole) e vítima (e.g., crenças negativas sobre si, baixa habilidade social, isolamento e clima escolar ruim; Cook, Willians, Guerra, Kim, \& Sadek, 2010; Fossati, Borroni, \& Maffei, 2012; Lucia, 2016; Melander, Hartshorn, \& Whitbeck, 2013; Salmivalli, 2010; Tani, Greenman, Schneider, \& Fregoso, 2003).

Logo, nota-se que os estudos vêm tendo em conta uma miríade de variáveis para compreender a predisposição de crianças e adolescentes para serem perpetradores ou vítimas de bullying, não se limitando àquelas mais individuais, como os traços de personalidade, tendo em conta igualmente a importância do contexto para a prática e desenvolvimento do bullying (Bowes et al., 2009; Lam, Law, Chan, Wong, \& Zhang, 2014;
Lee, 2009). Além disso, estima-se que o sexo e a idade dos escolares são características individuais que podem ter influência no envolvimento em episódios de bullying (Lee, 2009).

Concretamente, verifica-se a prevalência de homens tanto na categoria de vítima como de agressor (Carlyle \& Steinman, 2007; Lucia, 2016; Malta et al., 2014; Sourander, Hestelä, Helenius, \& Piha, 2000). Por exemplo, Malta et al. ( $N=109.104$ escolares brasileiros) verificaram que $7,9 \%$ dos homens e $6,5 \%$ das mulheres envolveram-se em bullying no papel de vítima, ao passo que $26,1 \%$ dos homens e $16 \%$ das mulheres o fizeram no papel de agressores. Ressalta-se, ainda, que a intensidade do bullying pode variar de acordo com a idade dos escolares, sendo que no início da adolescência há um pico na busca por popularidade, onde a prática do bullying pode ser usada para reforçar ou aumentar o próprio status (LaFontana \& Cillessen, 2010). A propósito, em revisão sistemática, Álvarez-García, García e Núñez (2015) observaram que a prática do bullying e a idade possuem um padrão de correlações curvilinear, ou seja, a probabilidade de praticar bullying aumenta seguidamente até os 14 anos, sendo que a partir desta idade, a probabilidade de agredir os pares diminui. Portanto, considerar variáveis demográficas pode ser importante para o entendimento do bullying, conjeturando que sua interação pode mudar as relações entre uma variável preditora e os comportamentos de bullying.

Diante do exposto, parece pertinente conhecer preditores potenciais do envolvimento em comportamentos de bullying, seja no papel de agressor ou vítima. Não obstante, ressalta-se que no presente estudo o foco são as pessoas envolvidas em bullying no papel de agressor. A propósito, estudos apontam que este grupo apresenta deficiências empáticas, desempenho acadêmico insatisfatório e que avaliam negativamente o clima escolar, além de serem expostos a múltiplas formas de violência doméstica (Del Rey et al., 2016; Lucas, Jernbro, Tindberg, \& Janson, 2015; Nansel et al., 2001). Ademais, praticar bullying na infância está relacionado a graves problemas na adultez, como personalidade antissocial, abu- 
so de substâncias e transtornos de ansiedade e depressão (Sourander et al., 2007).

Nesta direção, conhecer fatores relacionados a prática do bullying possibilita a identificação precoce de crianças mais propensas a agredirem seus pares. Tal aspecto é fundamental na medida em que auxilia no delineamento de intervenções que foquem na redução do bullying, fomentando um ambiente escolar propício para o aprendizado, além de ser importante na prevenção de futuros comportamentos criminais, de modo que praticar bullying na infância é um preditor de delinquencia na idade adulta (Álvarez-García et al., 2015; Bender \& Lösel, 2011).

Dentre as variáveis de cunho psicológico que podem contribuir para a explicação deste tipo de agressão entre pares, destacam-se os valores humanos. Estes têm um forte componente social, evidenciando a centralidade da socialização no processo de sua transmissão, algo importante de se ter em conta no estudo do bullying, uma vez que a influência familiar e dos pares pode incentivar a prática de tais condutas antissociais (Knafo, 2003; Ribeiro, 2007). Portanto, considerando que os valores socializados em determinados contextos podem ajudar a explicar a prática de bullying, no tópico a seguir a relação entre os dois construtos será explorada.

\section{Valores e Bullying}

A concepção de valores humanos adotada no presente estudo é oriunda da Teoria Funcionalista (Gouveia, 2013; Gouveia, Milfont, \& Guerra, 2014), uma perspectiva individual que considera os valores como aspectos psicológicos que possuem duas funções: guiar as ações humanas e representar cognitivamente suas necessidades. Do cruzamento das funções derivam seis subfunções valorativas, descritas a seguir, indicando-se entre parênteses três valores específicos que servem para representá-las:

Subfunção Experimentação (emoção, prazer e sexualidade). Estes valores descrevem pessoas pautadas pela busca de sensações; os valores que a compõem promovem uma maior facilidade de mudança e inovação nas estruturas sociais.

Subfunção Realização (êxito, poder e prestígio). São valores típicos de pessoas que buscam realizações materiais, procurando ser práticas em seus comportamentos e suas decisões, visando seu sucesso pessoal.

Subfunção Existência (estabilidade pessoal, saúde e sobrevivência). O propósito principal destes valores é garantir as condições básicas para a sobrevivência biológica e psicológica do individuo, o que é compatível com perseguir valores pessoais e sociais.

Subfunção Suprapessoal (beleza, conhecimento e maturidade). Seus valores representam necessidades estéticas, de cognição e autorrealização, traduzindo um entendimento maduro da vida e acentuando a relevância de princípios gerais e abstratos, coerente com orientações pessoais e sociais.

Subfunção Interativa (afetividade, apoio social e convivência). Tais valores representam necessidades de pertença, amor e afiliação, proporcionando o estabelecimento e a manutenção das relações interpessoais por parte da pessoa, que prioriza os demais.

Subfunção Normativa (obediência, religiosidade e tradição). Estes valores refletem a importância de preservar a cultura e as normas sociais, onde a obediência é valorizada acima de tudo, evidenciando a hierarquia social como princípio-reitor.

Considerando a descrição destas subfunções e as evidências que indicam que a prática do bullying está relacionada com elevado status e dominância frente aos pares (Pouwels, Lansu, \& Cillessen, 2016; Salmivalli, 2010), é possível hipotetizar que os envolvidos em comportamentos de bullying, no papel de agressor, priorizem valores de realização, visto que estes caracterizam pessoas que buscam o poder e o reconhecimento, podendo-se utilizar de agressões como um meio para alcançar posições de liderança (Benish-Weisman, 2015; LaFontana \& Cillessen, 2010; Schumann, Craig, \& Rosu, 2014). Por outro lado, valores sociais, especificamente os interativos, que descrevem aquelas pessoas pautadas em interesses sociais, que valorizam o estabelecimento de laços afetivos duradouros, podem se configurar como um fator de proteção contra a prática do bullying (Gouveia, 2013). 
De fato, estas presumíveis associações foram verificadas em estudos utilizando o modelo de Schwartz (1992), quando se constatou correlação positiva do comportamento agressivo entre pares e os valores de autopromoção (poder e realização) e, em sentido oposto, com os de autotranscendência (universalismo e benevolência), que descrevem aqueles que preocupam-se com o bem-estar e interesse dos demais (Benish-Weisman, 2015; Knafo, 2003; Knafo, Daniel, \& Khoury-Kassabri, 2008; Menesini, Nocetini, \& Camodeca, 2013).

Em resumo, objetivou-se neste estudo conhecer o papel preditivo dos valores, desde de uma perspectiva funcionalista, com relação aos comportamentos de bullying, além de averiguar em que medida as variáveis sexo e idade do perpetrador podem moderar esta relação. Especificamente, duas hipóteses foram elaboradas a partir da literatura:

1. As pontuações dos participantes em valores de realização estarão positivamente correlacionadas com aquelas na medida de prática de bullying e

2. Aquelas em valores da subfunção interativa o farão inversamente com este tipo de prática agressiva.

Esclarece-se que as relações entre valores e bullying poderão ser moderadas por variáveis demográficas, a exemplo de idade e sexo, que, no caso dos valores humanos, parecem exercer efeitos diferentes (Feather, 2004; Gouveia, Vione, Milfont, \& Fischer, 2015), o que talvez reflita na explicação de práticas de bullying. Por exemplo, uma vez que os homens priorizam mais do que as mulheres valores de realização, além de apresentarem mais condutas agressivas instrumentais, como as do bullying (e.g., bater, humilhar, chutar; Wang, Iannotti, \& Nansel, 2009), isso faria mais provável esse comportamento entre eles. Por outro lado, como as mulheres pontuam mais do que os homens em valores interativos, estando menos predispostas a condutas agressivas instrumentais do que os homens, talvez elas se engajassem menos na prática do bullying. Não obstante, em razão da escassez de estudos sobre estas presumíveis moderações, decidiu-se não formular qualquer hipótese a respeito.

\section{Método}

\section{Participantes}

Contou-se com a participação de 300 estudantes da cidade de Parnaíba (PI), apresentando idade variando de 8 a 17 anos $(M=11,07 ; D P=$ $1,31)$, sendo a maioria do sexo feminino $(53,1 \%)$ e de escola particular $(50,5 \%)$. Tratou-se de uma amostra de conveniência (não probabilista), tendo participado aqueles que, quando presentes em sala de aula e inqueridos a colaborar, aceitaram voluntariamente a participar da pesquisa. Destaca-se que $15,8 \%$ dos participantes indicaram cometer bullying ao passo que $25,3 \%$ afirmaram serem vítimas deste tipo de agressão.

\section{Instrumentos}

Os participantes responderam um livreto que, além de apresentar perguntas demográficas ao final (idade, sexo e tipo de escola), reuniu os seguintes instrumentos:

Escala de Comportamentos de Bullying (ECB; Medeiros, Gouveia et al., 2015). Medida composta por 16 itens respondidos em escala de cinco pontos $(0=$ Nenhuma vez a $4=$ Quatro ou mais vezes por semana). Tais itens são respondidos tendo em conta a seguinte frase estímulo: "em minha escola, ultimamente, apresentei tais comportamentos em relação aos meus colegas". Este instrumento reúne quatro fatores, correspondendo às formas de expressão do fenômeno: bullying físico (e.g., Chutei ou dei pontapés em colegas), verbal (e.g., Apelidei colegas), relacional (e.g., Excluí e/ou convenci amigos a isolarem outros colegas) e cyberbullying (e.g., Publiquei, na internet, fotos com o objetivo de ridicularizar colegas). No presente estudo observou-se uma estrutura fatorial satisfatória (CFI $=0,93$; TLI $=0,92$; RMSEA $=0,02$ ), com índices de consistência interna $(\alpha)$ variando de 0,64 (bullying relacional) a 0,82 (cyberbullying).

Questionário dos Valores Básicos Infantil (QVB-I; Gouveia, Milfont, Soares, Andrade, \& Leite, 2011). Esta medida é composta por 18 itens ou valores específicos adaptados para crianças (e.g., Poder. Ser o(a) chefe ou líder do grupo de amigos(as); mandar nos(as) outros(as) 
colegas; e ter poder para decidir as coisas; Convivência. Conviver bem com familiares e vizinhos; ter amigos(as) no colégio ou no bairro; e participar de atividades e brincadeiras com os(as) colegas). Os itens são respondidos em escala de cinco pontos $(1=$ Nenhuma importância a 5 = Máxima importância), e distribuídos em seis fatores ou subfunções valorativas. Nesta ocasião, a estrutura fatorial apresentou índices de ajuste adequados (CFI $=0,96$; TLI $=0,95$; RMSEA $=0,04)$, sendo observado os seguintes coeficientes do alfa de Cronbach: experimentação $(\alpha=0,55)$, realização $(\alpha=0,59)$, existência $(\alpha=0,48)$, suprapessoal $(\alpha=0,40)$, interativa ( $\alpha$ $=0,51)$ e normativa $(\alpha=0,51)$.

\section{Procedimento}

Após parecer favorável do comitê de ética em pesquisa da Universidade Federal do Piauí (CAAE: 0193.0.045.000-11), entrou-se em contato com os responsáveis pelas instituições de ensino com o intuito de solicitar a autorização para a realização da coleta dos dados. Por se tratar de uma amostra composta por menores, após a autorização dos diretores das escolas, foi enviado aos pais e responsáveis um termo de responsabilidade, onde deveriam dar ciência do estudo, expressando sua autorização a que o menor fizesse parte do estudo. A coleta dos dados foi realizada em ambiente coletivo (sala de aula), mas os questionários foram respondidos de forma individual. No momento da coleta, contou-se com a colaboração de dois aplicadores, devidamente aptos a dirimir quaisquer dúvidas sobre o instrumento. Destaca-se que a todos foi informado que a participação seria anônima e voluntária, não implicando qualquer dano ao participante, que poderia desistir da pesquisa a qualquer momento, sem prejuízos. Em média, foram necessários 30 minutos para concluir a participação no estudo.

\section{Análise dos Dados}

Os dados foram analisados por meio dos pacotes estatísticos PASW (versão 18) e R (R Core Team, 2015). Com o primeiro calcularam-se estatísticas descritivas, análises de correla- ção de Pearson, e análise de regressão múltipla para verificar em que medida os valores estão relacionados e predizem os comportamentos de bullying; a análise de regressão hierárquica foi também empregada com o fim de testar o papel moderador do sexo e da idade dos participantes.

Com o software R, por meio do pacote lavaan (Rosseel, 2012), verificou-se a adequação das medidas utilizadas, empregando o estimador Weighted Least Squares Means and Variance Adjusted (WLSMV), considerando os seguintes índices de ajuste: Comparative Fit Index (CFI), Tucker-Lewis Index (TLI) e Root Mean-Square Error of Approximation (RMSEA). Para considerar o modelo aceitável, os valores de CFI e TLI devem ser acima de 0,90, preferencialmente acima de 0,95 (Kline, 2016) e o RMSEA abaixo de 0,08 (Brown, 2006; Kline, 2016).

\section{Resultados}

Inicialmente, calcularam-se correlações de Pearson com o fim de conhecer em que medida e direção as seis subfunções valorativas se correlacionariam com as formas de expressão do bullying e sua pontuação total (Tabela 1). Avaliando o padrão de correlações $(p<0,05)$, observa-se que os valores de realização se correlacionaram positivamente com os quatro tipos de bullying, ao passo que a subfunção interativa o fez negativamente com três formas de expressão do bullying (físico, relacional e cyberbullying). Além destas subfunções, a suprapessoal, que como a anterior representa um motivador humanitário, idealista, também se correlacionou inversamente com duas dimensões da medida de bullying (relacional e cyberbullying). A pontuação total da ECB se correlacionou com as subfunções realização $(r=0,28 ; p<0,01)$, interativa $(r=-0,14 ; p<0,01)$ e suprapessoal $(r=$ $-0,12 ; p<0,05)$.

Passo seguinte, por meio de análise de regressão múltipla (método stepwise), verificou-se quais subfunções valorativas prediriam os comportamentos de bullying. Considerou-se a pontuação total da ECB como variável critério por conta das dimensões específicas do bullying se correlacionarem de forma consistente com 


\section{Tabela 1}

Correlações e Regressão Hierárquica das Subfunções Valorativas e Fatores de Bullying

\begin{tabular}{|c|c|c|c|c|c|c|}
\hline \multirow{2}{*}{$\frac{\text { Correlações }}{\text { Valores Humanos }}$} & \multicolumn{6}{|c|}{ Comportamentos de Bullying } \\
\hline & Total & Físico & Verbal & Rela & & Cyberbullying \\
\hline Experimentação & $-0,02$ & $-0,03$ & 0,01 & & & $-0,06$ \\
\hline Realização & $0,28 * *$ & $0,18 * *$ & $0,21 * *$ & & & $0,12^{*}$ \\
\hline Suprapessoal & $-0,12 *$ & $-0,09$ & $-0,08$ & & & $-0,13^{*}$ \\
\hline Existência & $-0,08$ & $-0,04$ & 0,01 & & & $-0,12 *$ \\
\hline Interativa & $-0,16^{* *}$ & $-0,14^{* *}$ & $-0,08$ & & & $-0,14^{* *}$ \\
\hline Normativa & $-0,07$ & $-0,05$ & $-0,06$ & & & $-0,09$ \\
\hline \multicolumn{7}{|l|}{ Regressões } \\
\hline Realização x Idade & $\beta$ & $t$ & \multicolumn{2}{|c|}{ Interativa $\mathrm{x}$ Idade } & $\beta$ & $t$ \\
\hline zRealização & $0,28 * *$ & 4,53 & \multicolumn{2}{|c|}{ zInterativa } & $-0,18^{* *}$ & $-2,86$ \\
\hline zIdade & $-0,03$ & $-0,54$ & \multicolumn{2}{|c|}{ zIdade } & $-0,04$ & $-0,60$ \\
\hline Interação & $-0,01$ & $-0,14$ & \multicolumn{2}{|c|}{ Interação } & 0,04 & 0,64 \\
\hline Realização x Sexo & \multicolumn{6}{|c|}{ Interativa $\mathrm{x}$ Sexo } \\
\hline C_Realização & 0,34 & 1,65 & \multicolumn{2}{|c|}{ C_Interativa } & $-0,06$ & $-0,33$ \\
\hline Sexo & $-0,21 * *$ & $-3,51$ & \multicolumn{2}{|c|}{ Sexo } & $-0,20 * *$ & $-3,16$ \\
\hline Interação & $-0,07$ & $-0,37$ & \multicolumn{2}{|c|}{ Interação } & $-0,08$ & $-0,42$ \\
\hline
\end{tabular}

Nota. ${ }^{*} p<0,05, * * p<0,01$ (teste uni-caudal).

as subfunções realização e interativa, tal como hipotetizado, além destes fatores de bullying estarem relacionados entre si, justificando o cômputo de um fator geral. Os resultados reforçaram os papéis preditores dos valores das subfunções realização $(\beta=0,32, \mathrm{~B}=0,14, \mathrm{IC} 95 \%=$ $0,09 / 0,20, p<0,001)$ e interativa $(\beta=-0,21, \mathrm{~B}$ $=-0,14$, IC95\% $=-0,23 /-0,06, p<0,001)$. Estas duas subfunções apresentaram $R=0,37$, explicando conjuntamente $14 \%\left(R^{2}\right)$ da variância das pontuações da medida de bullying $(p<0,001)$.

Posteriormente, realizaram-se análises de regressão hierárquica para verificar se a idade e o sexo moderariam as relações supracitadas (valores predizendo bullying; Tabela 1). Inicialmente, seguindo o procedimento de Miles e Shevlin (2001) para preditores contínuos, padronizou-se (z) a variável realização e a idade, multiplicando uma pela outra, computando-se assim o termo de interação. Em seguida, entrou-se com a subfunção realização e a idade padronizadas no primeiro passo da regressão hierárquica, introdu- zindo a interação entre ambas no segundo passo desta análise. Não se verificou qualquer predição significativa do termo de interação, descartando a possível moderação. Testou-se ainda se a idade moderaria a relação entre a subfunção interativa e os comportamentos de bullying, seguindo-se o procedimento anteriormente descrito. No caso, apenas a subfunção interativa predisse os comportamentos de bullying, mas não o termo de interação. Deste modo, não se configurou o papel moderador da idade.

Buscou-se verificar se o sexo moderaria a relação entre a subfunção realização e os comportamentos de bullying. No caso, seguindo o procedimento de Miles e Shevlin (2001) para um preditor contínuo e uma moderadora categórica, centralizou-se a variável realização, multiplicando-a pelo sexo para criar o termo de interação. Logo, entrou-se com as variáveis realização e sexo no primeiro passo na regressão hierárquica e o termo de interação no segundo passo. No entanto, não se verificou predição significativa 
deste termo. Portanto, o sexo não moderou a relação entre realização e bullying. Realizou-se o mesmo procedimento para verificar a moderação do sexo na relação entre a subfunção interativa $\mathrm{e}$ os comportamentos de bullying, não sendo verificada predição do termo de interação, o que descarta o papel moderador do sexo neste contexto.

\section{Discussão}

O presente estudo teve como objetivo conhecer em que medida e direção os valores humanos se correlacionariam e prediriam os comportamentos de bullying, checando o papel moderador das variáveis sexo e idade dos respondentes. Confia-se que este objetivo tenha sido alcançado. Concretamente, foi possível verificar que os valores foram preditores de comportamentos típicos do bullying, algo congruente com os resultados encontrados em estudos prévios (Knafo, 2003; Knafo et al., 2008; Menesini et al., 2013).

Pareceu evidente, pois, que os perpetradores deste tipo de agressão são orientados pela busca de reconhecimento e poder, provavelmente procurando se impor por meio da agressão, em uma tentativa de serem respeitados por seus pares, assumindo uma posição de destaque e liderança (Benish-Weisman, 2015; LaFontana \& Cillessen, 2010; Schumann et al., 2014). Crianças e adolescentes que praticam bullying são percebidos por seus pares como populares, portanto, a agressão pode ser uma forma utilizada para alcançar status e uma posição social dominante (de Bruyn, Cillessen, \& Wissink, 2010; Morales, Yubero, \& Larrañaga, 2016; Pouwels et al., 2016). Deste modo, há que demandar algum cuidado em promover descontextualizadamente os valores de realização, fazendo perceber a importância também das relações interpessoais.

A propósito do anteriormente comentado, verificou-se que aquelas crianças que se orientam pela subfunção interativa, representada pelos valores específicos afetividade, apoio social e convivência, foram menos prováveis de se envolverem em comportamentos de bullying. Estes valores representam necessidades de pertença, amor e afiliação, proporcionando a manutenção e harmonia das relações interpessoais, promovendo condutas de respeito mútuo (Gouveia, 2003, 2013; Medeiros et al., 2012). Neste sentido, tais valores agem como um fator de proteção para os comportamentos de bullying, inibindo-os. Deste modo, parece relevante incentivar a transmissão destes valores por parte de pais e professores.

Diante deste cenário, parece evidente a importância da teoria funcionalista dos valores humanos (Gouveia, 2003, 2013; Gouveia et al., 2014) para compreender os principais resultados deste estudo. Como pode ser predito a partir da descrição de suas subfunções teorizadas, crianças que já desde tenra idade se orientam por êxito, poder e prestígio são mais propensas a envolverem-se em situaçoes de bullying no papel de agressores ou estariam mais dispostas a fazer valer seus interesses pessoais, impondo-se aos demais, o que pode acarretar em comportamentos de bullying nas escolas. Não se está afirmando categoricamente que estas pessoas seriam bullies, mas que a importância atribuída a esta subfunção agiria como um fator de risco para tais comportamentos. Portanto, aqueles que buscam realizações materiais e preferem agir de modo prático ao tomarem decisões e expressarem comportamentos estão mais predispostos a se envolverem em comportamentos discriminadores, em que o bulying é um dos tipos. Contrariamente, conferir importância aos valores humanitários, sobretudo de orientação social, como os interativos, promovem a aceitação do outro, sendo menos discriminador, além de evitar promover condutas que depreciem os demais. Portanto, parece claro o importante papel que os valores cumprem para o entendimento do bullying, explicando, nesta ocasião, 14\% de sua variância, valor superior ao de outros preditores de cunho individual (e.g., internalização [1,44\%], externalização [11,5\%] ou performance acadêmica [4,41\%]; Cook et al., 2010).

Fica evidente que as duas hipóteses deste estudo foram corroboradas. Entretanto, é importante assinalar que as relações entre valores e comportamentos de bullying não foram moderadas pelas variáveis sexo e idade dos participantes, como poderia ser inicialmente conjeturado a partir de estudos empíricos que citam 
o papel destas variáveis demográficas para o entendimento do bullying (Carlyle \& Steinman, 2007; LaFontana \& Cillessen, 2010; Sourander et al., 2000). Especificamente, não há que perder de vista que a variação da idade dos participantes, concentrada entre 10 a 12 anos $(77,6 \%$ da amostra), pode ter sido determinante para não configurar qualquer relação com o bullying. Nesta direção, em estudos futuros é importante considerar amostras mais heterogêneas quanto a idade, pois esta variável apresenta um padrão de correlações curvilinear com o envolvimento em bullying (Álvarez-García et al., 2015).

A propósito de possibilidades de estudos futuros, será relevante ter em conta outras variáveis preditoras do bullying, a exemplo dos estilos parentais, visto que é no contexto familiar que a criança tem suas primeiras oportunidades de socialização, constituindo-se um papel preponderante no comportamento que adotará e na sua interação com os demais (Ribeiro, 2007). Portanto, estima-se que a possível exposição de infantes a episódios de violência familiar, caracterizado muitas vezes por um estilo autoritário, é consistente com alto risco de envolvimento em comportamentos de bullying, pois esta passa a perceber a violência como um método aceitável para resolução de conflitos (Bauer et al., 2006; Bowes et al., 2009; Lucas et al., 2015). Nesta direção, é comum a presença de pais autoritários e superproterores na família de agressores, enquanto que os permissivos são mais típicos nas familias de vítimas (Baldry \& Farrington, 2000; Georgiou, 2008; Knafo, 2003).

Também será importante testar outros moderadores da relação entre valores e comportamentos de bullying. Concretamente, poder-se-á verificar em que medida os valores dos próprios pais das crianças agem como moderadores dessa relação. Também caberia considerar o fato de tais pais apoiarem a prática discriminatória de bullying, mostrando comportamentos discriminatórios em relação a grupos minoritários (e.g., gays, negros, gordos, etc.). Portanto, abrem-se diversas alternativas de pesquisas que consideram os valores como explicadores do bullying, mas poderão ser igualmente considerados outros construtos explicadores, a exemplo dos traços de personalidade e da crença no mundo justo. É possível que a naturalização deste tipo de comportamento agressivo seja mais comum entre aqueles com alta crença na ideia de que cada um tem o que merece, isto é, o mundo é essencialmente justo, pois assim justifica que grupos minoritários sejam discriminados e objeto de agressão (Uhlmann, Brescoll, \& Machery, 2010).

Por fim, cabe ressaltar potenciais limitações da pesquisa. Por exemplo, há que considerar que a amostra não foi probabilística, impossibilitando a generalização dos achados para além do escopo da pesquisa. Ressalta-se, ainda, o fato de terem sido empregas medidas de autorrelato, que podem ser influenciadas pela desejabilidade social dos participantes no momento de responder os questionários. Isso é particularmente possível em razão do tipo de construto que se está tentando explicar, isto é, o bullying, que é presumivelmente indesejável pela maior parte das pessoas.

Apesar das limitações expostas, os resultados encontrados indicam o importante papel dos valores para a compreensão do bullying, sendo que intervenções pautadas na promoção de valores podem contribuir para a redução de tais comportamentos agressivos entre os escolares. Concretamente, uma possibilidade de intervenção seria orientantar pais e professores a passar para as crianças a importância dos valores, principalmente aqueles de uma orientação social, que vem se mostrando de forma consistente como fatores de proteção contra atitudes e comportamentos problema (Formiga \& Gouveia, 2005; Medeiros, Pimentel, Monteiro, Gouveia, \& Medeiros, 2015; Pimentel, Gouveia, Medeiros, Santos, \& Fonseca, 2011).

\section{Referências}

Álvarez-García, D., García, T., \& Núñez, J. C. (2015). Predictors of school bullying perpetration in adolescence: A systematic review. Aggression and Violent Behavior, 23, 126-136.

Arseneault, L., Walsh, E., Trzesniewski, K., Newcombe, R., Caspi, A., \& Moffitt, T. E. (2006). Bullying victimization uniquely contributes to adjustment problems in young children: A nationally representative cohort study. Pediatrics, 118, 130-138. 
Baldry, A. C., \& Farrington, D. P. (2000). Bullies and delinquents: Personal characteristics and parental styles. Journal of Community \& Applied Social Psychology, 10, 17-31.

Bauer, N. S., Herrenkohl, T. I., Lozano, P., Rivara, F. P., Hill, K. G., \& Hawkins, J. D. (2006). Childhood bullying involvement and exposure to intimate partner violence. Pediatrics, 118 , 235-242.

Bender, D., \& Lösel, F. (2011). Bullying at school as a predictor of delinquency, violence and other anti-social behaviour in adulthood. Criminal Behaviour and Mental Health, 21, 99-106.

Benish-Weisman, M. (2015). The interplay between values and aggression in adolescence: A longitudinal study. Developmental Psychology, 51, 677-687.

Blank, D., \& Liberal, E. F. (2005). O pediatra e as causas externas de morbimortalidade. Jornal de Pediatria, 81, 119-122.

Bowes, L., Arseneault, L., Maughan, B., Taylor, A., Caspi, A., \& Moffitt, T. E. (2009). School, neighborhood, and family factors are associated with children's bullying involvement: A nationally representative longitudinal study. Journal of the American Academy of Child \& Adolescent Psychiatry, 48, 545-553.

Brown, T. A. (2006). Confirmatory factor analysis for applied research. New York: The Guilford Press.

Carlyle, K. E., \& Steinman, K. J. (2007). Demographic differences in the prevalence, co-occurrence, and correlates of adolescent bullying at school. Journal of School Health, 77, 623-629.

Cook, C. R., Willians, K. R., Guerra, N. G., Kim, T. E., \& Sadek, S. (2010). Predictors of bullying and victimization in childhood and adolescence: A meta-analytic investigation. School Psychology Quarterly, 25, 65-83.

Costa, M. R., Xavier, C. C., Andrade, A. C. S., Proietti, F. A., \& Caiaffa, W. T. (2015). Bullying among adolescents in a Brazilian urban center - "Health in Beagá" study. Revista de Saúde Pública, 49, 1-10.

Craig, W., Harel-Fisch, Y., Fogel-Grinvald, H., Dostaler, S., Hetland, J., Simons-Morton, B., ...Pickett, W. (2009). A cross-national profile of bullying and victimization among adolescents in 40 countries. International Journal of Public Health, 54, 216-224.
De Bruyn, E. H., Cillessen, A. H., \& Wissink, I. B. (2010). Associations of peer acceptance and perceived popularity with bullying and victimization in early adolescence. The Journal of Early Adolescence, 30, 543-566.

Del Rey, R., Lazuras, L., Casas, J. A., Barkoukis, V., Ortega-Ruiz, R., \& Tsorbatzoudis, H. (2016). Does empathy predict (cyber) bullying perpetration, and how do age, gender and nationality affect this relationship? Learning and Individual Differences, 45, 275-281.

Farrington, D. P. (1993). Understanding and preventing bullying. In M. Tommy \& N. Morris, Crime and justice. Chicago, IL: University of Chicago Press.

Farrington, D. P., \& Ttofi, M. M. (2011). Bullying as a predictor of offending, violence and later life outcomes. Criminal Behaviour and Mental Health, 21, 90-98.

Feather, N. T. (2004). Value correlates of ambivalent attitudes toward gender relations. Personality and Social Psychology Bulletin, 30, 3-12.

Fleming, L. C., \& Jacobsen, K. H. (2009). Bullying and symptoms of depression in Chilean middle school students. Journal of School Health, 79, 130-137.

Formiga, N. S., \& Gouveia, V. V. (2005). Valores humanos e condutas anti-sociais e delitivas. Psicologia: Teoria e Prática, 7, 134-170.

Fossati, A., Borroni, S., \& Maffei, C. (2012). Bullying as a style of personal relating: Personality characteristics and interpersonal aspects of self-reports of bullying behaviours among Italian adolescent high school students. Personality and Mental Health, 6, 325-339.

Geel, M. V., Vedder, P., \& Tanilon, J. (2014). Relationship between peer victimization, cyberbullying, and suicide in children and adolescents: A meta-analysis. JAMA Pediatrics, 168 , 435-442.

Georgiou, S. N. (2008). Parental style and child bullying and victimization experiences at school. Social Psychology of Education, 11, 213-227.

Gouveia, V. V. (2003). A natureza motivacional dos valores humanos: Evidências acerca de uma nova tipologia. Estudos de Psicologia (Natal), 8, 431-443.

Gouveia, V. V. (2013). Teoria funcionalista dos valores humanos: Fundamentos, aplicações e perspectivas. São Paulo, SP: Casa do Psicólogo. 
Gouveia, V.V., Milfont, T. L., \& Guerra, V. M. (2014). Functional theory of human values: Testing its content and structure hypotheses. Personality and Individual Differences, 60, 41-47.

Gouveia, V. V., Milfont, T. L., Soares, A. K. S., Andrade, P. R., \& Leite, I. L. (2011). Conhecendo os valores na infância: Evidências psicométricas de uma medida. Psico, 42, 106-115.

Gouveia, V. V., Vione, K. C., Milfont, T. L., \& Fischer, R. (2015). Patterns of value change during the life span: Some evidence from a functional approach to values. Personality and Social Psychology Bulletin, 41, 1276-1290.

Hertz, M. F., Donato, I., \& Wright, J. (2013). Bullying and suicide: A public health approach. Journal of Adolescent Health, 53(Suppl.), S1-S3.

Kim, Y. S., Koh, Y. J., \& Leventhal, B. (2005). School bullying and suicidal risk in Korean middle school students. Pediatrics, 115, 357-363.

Kline, R. B. (2016). Principles and practice of structural equation modeling ( $4^{\text {th }}$ ed.). New York: Guilford Press.

Klomek, A. B., Sourander, A., \& Gould, M. (2010). The association of suicide and bullying in childhood to young adulthood: A review of cross-sectional and longitudinal research findings. The Canadian Journal of Psychiatry, 55, 282288.

Knafo, A. (2003). Authoritarians, the next generation: Values and bullying among adolescent children of authoritarian fathers. Analyses of Social Issues and Public Policy, 3, 199-204.

Knafo, A., Daniel, E., \& Khoury-Kassabri, M (2008). Values as protective factors against violent behavior in Jewish and Arab high schools in Israel. Child Development, 79, 652-667.

LaFontana, K. M., \& Cillessen, A. H. N. (2010). Developmental changes in the priority of perceived status in childhood and adolescence. Review of Social Development, 19, 130-147.

Lam, S. F., Law, W., Chan, C.-K., Wong, B. P. H., \& Zhang, X. (2014). A latent class growth analysis of school bullying and its social context: The self-determination theory perspective. School Psychology Quarterly, 30, 75-90.

Lee, C. H. (2009). Personal and interpersonal correlates of bullying behaviors among Korean middle school students. Journal of Interpersonal Violence, 25, 152-176.
Lopes Neto, A. A. (2005). Bullying: Comportamento agressivo entre estudantes. Jornal de Pediatria, 81, 164-172.

Lucas, S., Jernbro, C., Tindberg, Y., \& Janson, S. (2015). Bully, bullied and abused. Associations between violence at home and bullying in childhood. Scandinavian Journal of Public Health, 44, 1-9.

Lucia, S. (2016). Correlates of bullying in Switzerland. European Journal of Criminology, 13, 50-66.

Malta, D. C., Porto, D. L., Crespo, C. D., Silva, M. M. A., Andrade, S. S. C., Mello, F. C. M., Monteiro, R., \& Silva, M. A. I. (2014). Bullying em escolares brasileiros: Análise da Pesquisa Nacional de Saúde do Escolar (PeNSE 2012). Revista Brasileira de Epidemiologia, 17(Supl. 1), 92-105.

Medeiros, E. D., Gouveia, V. V., Gusmão, E. E. S., Milfont, T. L., Fônseca, P. N., \& Aquino, T. A. A. (2012). Teoria funcionalista dos valores: Evidências de sua adequação no contexto paraibano. Revista de Administração Mackenzie, 13, 18-44.

Medeiros, E. D., Gouveia, V. V., Monteiro, R. P., Silva, P. G. N., Lopes, B. J., Medeiros, P. C. B., \& Silva, E. S. (2015). Escala de Comportamentos de Bullying (ECB): Elaboração e evidências psicométricas. Psico-USF, 20, 385-397.

Medeiros, E. D., Pimentel, C. E., Monteiro, R. P., Gouveia, V. V., \& Medeiros, P. C. B. (2015). Valores, atitudes e uso de bebidas alcoólicas: Proposta de um modelo hierárquico. Psicologia: Ciência e Profissão, 35, 841-854.

Melander, L. A., Hartshorn, K. J. S., \& Whitbeck, L. B. (2013). Correlates of bullying behaviors among a sample of North American Indigenous adolescents. Journal of Adolescence, 36, 675684.

Menesini, E., Nocetini, A., \& Camodeca, M. (2013). Morality, values, traditional bullying, and cyberbullying in adolescence. British Journal of Developmental Psychology, 31, 1-14.

Miles, J. N. V., \& Shevlin, M. E. (2001). Applying regression and correlation: A guide for students and researchers. London: Sage.

Morales, J. F., Yubero, S., \& Larrañaga, E. (2016). Gender and bullying: Application of a three-factor model of gender stereotyping. Sex Roles, 74, 169-180. 
Nansel, T. R., Overpeck, M., Pilla, R. S., Ruan, W. J., Simons-Morton, B., \& Scheidt, P. (2001). Bullying behaviors among US youth: Prevalence and association with psychosocial adjustment. Jama, 285, 2094-2100.

Oliveira, W. A., Silva, M. A. I., Silva, J. L., Mello, F. C. M., Prado, R. R., \& Malta, D. C. (2016). Associações entre a prática do bullying e variáveis individuais e de contexto na perspectiva dos agressores. Jornal de Pediatria, 92, 32-39.

Olweus, D. (1993). Bullyng at school: What we know and what we can do. Cambridge, MA: Blackwell.

Perren, S., \& Alsaker, F. D. (2006). Social behavior and peer relationships of victims, bully-victims, and bullies in kindergarten. Journal of Child Psychology and Psychiatry, 47, 45-57.

Pimentel, C. E., Gouveia, V. V., Medeiros, E. D., Santos, W. S., \& Fonseca, P. N. (2011). Explicando atitudes frente à maconha $\mathrm{e}$ comportamentos antissociais: O papel dos valores e grupos normativos. In S. C. S. Fernandes, C. E. Pimentel, V. V. Gouveia, \& J. L. A. Estramiana (Eds.), Psicologia social: Perspectivas atuais e evidencias empíricas (pp. 13-23). São Paulo, SP: Casa do Psicólogo.

Pouwels, J. L., Lansu, T. A., \& Cillessen, A. H. (2016). Participant roles of bullying in adolescence: Status characteristics, social behavior, and assignment criteria. Aggressive Behavior, 42, 239-253.

R Core Team. (2015). R: A language and environment for statistical computing. Vienna, Austria: R Foundation for Statistical Computing. Retrieved from http://www.R-project.org/

Reed, K. P., Nugent, W., \& Cooper, R. L. (2015). Testing a path model of relationships between gender, age, and bullying victimization and violent behavior, substance abuse, depression, suicidal ideation, and suicide attempts in adolescents. Children and Youth Services Review, 55, 128-137.

Ribeiro, A. T. M. (2007). O bullying em contexto escolar: Estudo de caso. Dissertação de mestrado não publicada, Universidade Portucalense, Porto, Portugal.

Roh, B. R., Yoon, Y., Kwon, A., Oh, S., Lee, S. I., Ha, K., ...Hong, H. J. (2015). The structure of co-occurring bullying experiences and associations with suicidal behaviors in Korean adolescents. PloS one, 10, 1-14.
Rosseel, Y. (2012). lavaan: An R Package for Structural Equation Modeling. Journal of Statistical Software, 48, 1-36.

Salmivalli, C. (2010). Bullying and the peer group: A review. Agression and Violent Behavior, 15, 112-120.

Schwartz, S. H. (1992). Universal in the content and structure of values: Theoretical advances and empirical tests in 20 countries. In M. P. Zanna (Ed.), Advanced in experimental social psychology (pp. 1-65). New York: Academic Press.

Smith, P. K., \& Morita, Y. (1999). The nature of school bullying: A cross-national perspective. London: Routledge.

Sourander, A., Hestelä, L., Helenius, H., \& Piha, J. (2000). Persistence of bullying from childhood to adolescence - A longitudinal 8-year follow-up study. Child Abuse \& Neglect, 24, 873-881.

Sourander, A., Jensen, P., Rönning, J. A., Elonheimo, H., Niemela, S., Helenius, H., ...Almqvist, F. (2007). Childhood bullies and victims and their risk of criminality in late adolescence. Archives of Pediatrics \& Adolescent Medicine, 161, 546552.

Schumann, L., Craig, W., \& Rosu, A. (2014). Power differentials in bullying: Individuals in a community context. Journal of Interpersonal Violence, 29, 846-865.

Tani, F., Greenman, P. S., Schneider, B. H., \& Fregoso, M. (2003). Bullying and the big five: A study of childhood personality and participant roles in bullying incidents. School Psychology International, 24, 131-146.

Uhlmann, E. L., Brescoll, V. L., \& Machery, E. (2010). The motives underlying stereotype-based discrimination against members of stigmatized groups. Social Justice Research, 23, 1-16.

Wang, J., Iannotti, R. J., \& Nansel, T. R. (2009). School bullying among adolescents in the United States: Physical, verbal, relational and cyber. Journal of Adolescent Health, 45, 368-375

Recebido: 16/02/2016

$1^{a}$ revisão: $24 / 05 / 2016$

$2^{a}$ revisão: $17 / 06 / 2016$

$2^{a}$ revisão: $11 / 07 / 2016$

Aceite final: 12/07/2016 